\title{
THE CHARACTERIZATION OF METAMATERIALS IN VIRTUAL TESTING: MACROSCOPIC EFFECTIVE PROPERTIES FOR STRUCTURAL APPLICATIONS
}

\section{IVÁN MARÍN GONZÁLEZ*1, GONZALO VERA RODRÍGUEZ ${ }^{1}$, LUIS SAUCEDO-MORA ${ }^{1,3}$, MIGUEL ÁNGEL SANZ GÓMEZ ${ }^{1}$}

\author{
${ }^{1}$ E.T.S. de Ingeniería Aeronáutica y del Espacio. Universidad Politécnica de Madrid \\ Pza. Cardenal Cisneros 3, 28040-Madrid, Spain \\ info.aeroespacial@upm.es, https://www.etsiae.upm.es \\ ${ }^{2}$ Dept. of Mechanical and Aerospace Engineering. Herbert Wertheim College of Engineering, \\ University of Florida \\ Gainesville, FL 32611, USA \\ chair@mae.ufl.edu,https://www.eng.ufl.edu/academics/schools-departments/mae \\ ${ }^{3}$ Dept. of Materials, University of Oxford \\ Parks Road, OX1 3PH, Oxford, UK \\ enquiries@materials.ox.ac.uk, https://www.materials.ox.ac.uk/
}

\begin{abstract}
Authors : ivan.marin.gonzalez@alumnos.upm.es, gonzalo.vera.rodriguez@alumnos.upm.es, luis.saucedo@upm.es, fco.montans@upm.es,miguelangel.sanz@upm.es
\end{abstract}

Key words: Metamaterial, virtual testing, finite element, additive manufacturing

\begin{abstract}
Metamaterials research and, moreover, their obtaining by additive manufacturing is trendy because of their potential industrial applications, since lattice structures can save weight when integrated in larger parts. For this reason, it is important to draw these new material properties in order to compare them with traditional structures obtained by classic manufacturing methods. In this work we present a numerical procedure to determine the mechanical properties of a metamaterial based on periodic cells. To date, for these materials there are no standard procedures to test and validate their structural properties, and specifically to determine their mechanical bending behaviour. We apply a virtual testing procedure using finite elements to determine the homogeneous elastic modulus, Young's modulus and shear modulus, using a set of modified bending virtual test, based on the three point bending and the four point bending test [1]. Theses physical testing procedures are common in sandwich composite materials [2], and can be apply to metamaterial testing taking into account some geometrical and material aspects to determine the proper parameters for testing. The results obtained are macroscopically independent on the number of elemental cells used in the metamaterial, and permit us to consider the intrinsic defects that may appear in the 3D additive manufacturing materials.
\end{abstract}




\section{INTRODUCTION}

Metamaterials are not something new to nature, bones or honeycombs, wood or coral are an example of cellular materials. These metamaterials are formed thanks to the repetition of a representative structure called a unit cell. They can also be generated from a replication pattern wrapped in a geometric shape. In one way or another, there is a difference between the behavior that a single cell can have, to that obtained by repeating a large number of them. This strategy opens the door to new areas of knowledge within the world of materials, which thanks to new additive manufacturing techniques and the advancement of technology, are now explorable. The union of finite element modeling techniques together with an experimental testing methodology that can now be carried out with the development of manufacturing processes can result in materials with new characteristics that the industry can take advantage of.

Among its possible applications, the biomedical world seems to be able to benefit greatly from this type of materials, creating prostheses with unique deformation capabilities, in acoustics, manipulating the speed of the wave through the material, also in heat transfer, cell geometry can improve the performance of these systems by increasing the ratio of transfer area to total volume.

Because of these facts, we seek the possible structural application of these materials. Generally there is a relationship between the resistance and density of the materials, where the densest are very resistant and vice versa. Metamaterials can find high resistance relationships with lower densities, which would lead to a decrease in weight, which for structural applications is a factor of great importance and even differential in some sectors such as aeronautics.
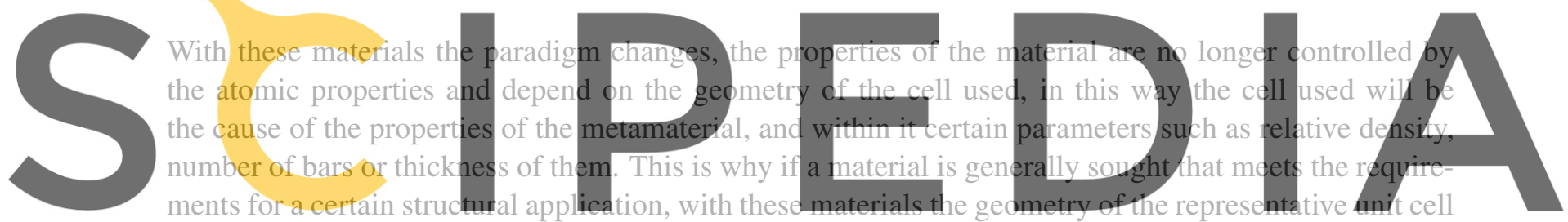
is also sought, which when replicated meets the needs sought.

Register for free at https//www.scipedia.com to download the version without the watermark In a way analogous to the development of composite materials, through a long process of testing, verification and standardization, it is possible that these materials perform the same tasks as other structural elements, introducing their advantages in them. For this moment to arrive, a path of research and development of models, techniques and results still has to be carried out.

\section{APPROACH TO CELL STRUCTURES}

The Finite Element Method (FEM) is the main tool used to carry out structural calculation tasks in any sector that is dedicated to it. Its development has facilitated that complex continuous problems could be addressed in a simple way. For this study, the FEM is used thanks to the professional Nastran software where virtual tests on metamaterials can be performed. The first objectives of this study consists on the familiarization with the behavior of this type of structures. Due to similarity to the metamaterials, the solutions provided in relation to Honeycombs will be studied.These materials are the most similar to the cell-like structures that will be seen later since they consist of a cellular structure of extruded hexagons in the perpendicular direction. 


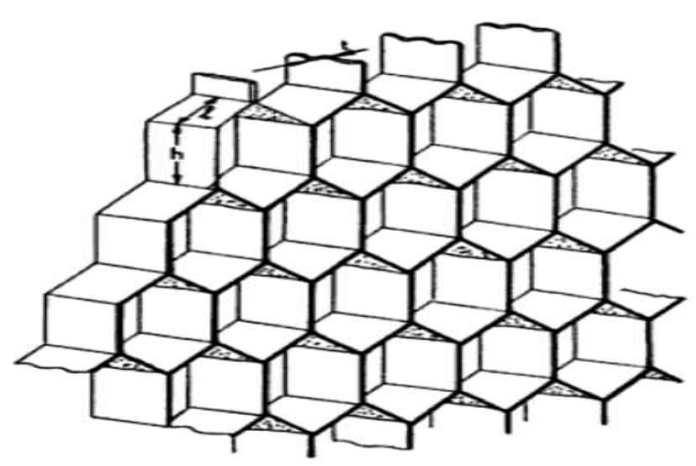

Figure 1: Honeycomb cell structure

For this study we seek to recreate the solutions provided in [1] using Nastran tools. What is tried to understand in this first approach is what number of cells is necessary, so that applying a distant load and not directly applied on the study cell, the displacement is as similar as possible to that described in the solutions in the book. With this, we seek to learn how the number of cells in the structure affects and reach the moment of independence, in which although there are more contiguous cells, the solution is still the same as that provided by the study document. In this way, in addition to the number of existing cells, they will affect how the boundary conditions and the load are imposed. The document does not establish anything about the boundary conditions, that the boundary conditio seen later, the form of $1 d$ of the best configuration. Below is the geometry of the hexagonal cell with the
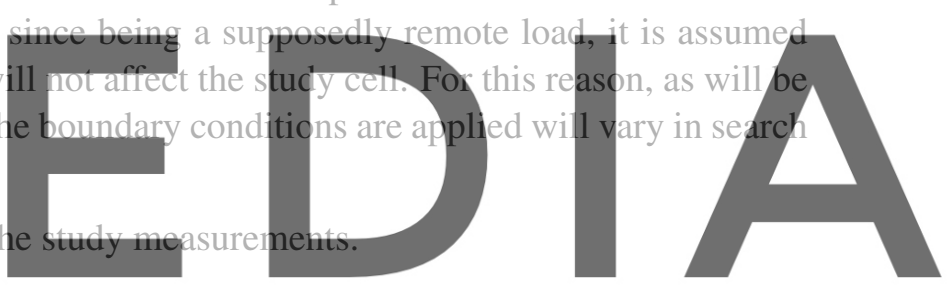

Register for free at https//www.scipedia.com to download the version without the watermark

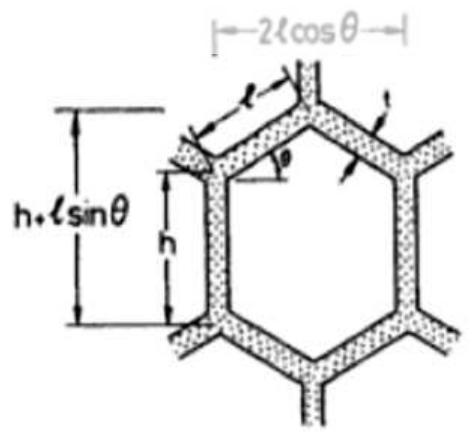

Figure 2: Hexagonal cell geometry

Where $\theta=30^{\circ}$ and $1=10 \mathrm{~mm}$. The thickness will vary between 1 and $0.5 \mathrm{~mm}$. The load cases that are 
studied are shown below.

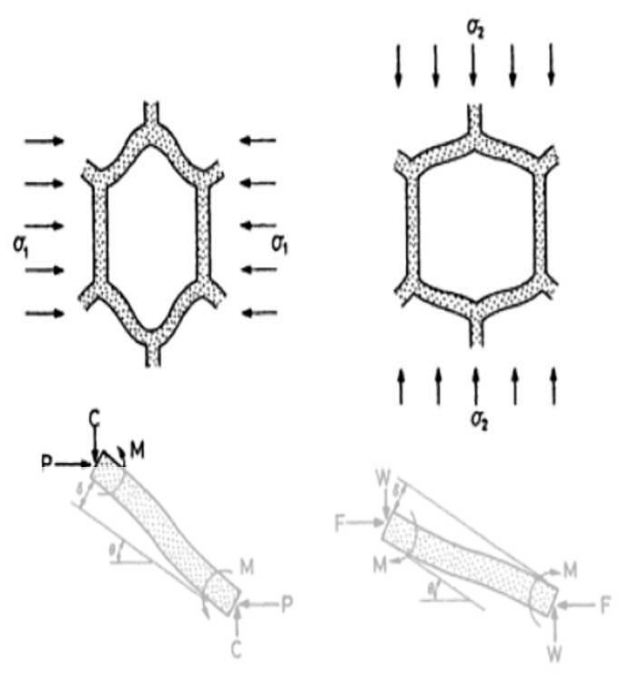

Figure 3: Hex Cell Load Cases

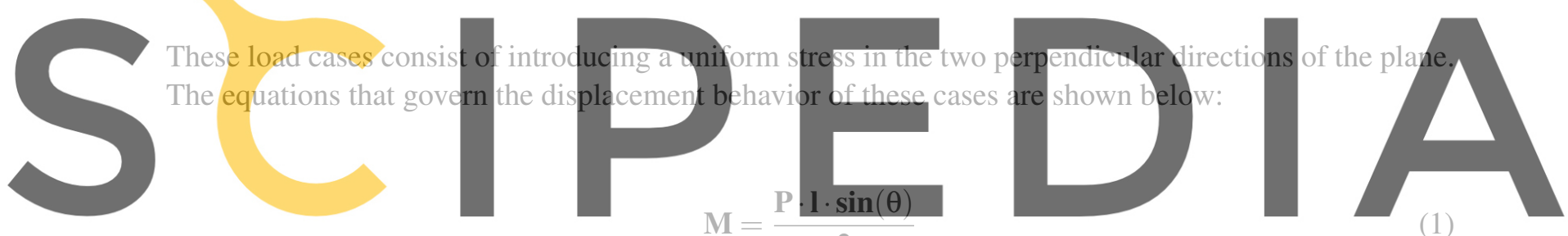
2

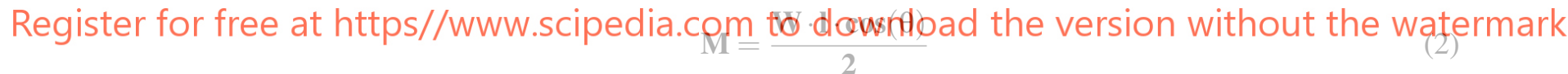

for the moments

$$
\begin{gathered}
\delta=\frac{\mathbf{P} \cdot \mathbf{l}^{3} \cdot \sin (\theta)}{12 \cdot \mathbf{E}_{\mathrm{s}} \cdot \mathbf{I}} \\
\delta=\frac{\mathrm{W} \cdot \mathbf{l}^{3} \cdot \cos (\theta)}{12 \cdot \mathbf{E}_{\mathrm{s}} \cdot \mathbf{I}}
\end{gathered}
$$

for displacements. In these relationships $E_{s}$ is the elastic modulus of the substrate material, which will be Aluminum with $\mathrm{E}=70 \mathrm{GPa}, v=0.33$ and the moment of inertia for constant thickness $\mathrm{I}=\mathrm{b} \cdot t^{3} / 12$. 
The operational form of calculation will be as follows. In Patran, the study model will be created with a load that manages to emulate a uniform tension that reaches the study cell. The Nastran output will read the displacement of the study node. To compare it with the result of the equations, the moment of the element that contains the node will be read, with equations (1) and (2) the force that reaches the node will be obtained and using the relations (3) and (4) the displacement that the equations stones is achieved. In order to compare it, the error is defined as follows:

$$
\text { error }=\frac{\mathbf{u}_{\text {Nastran }}-\mathbf{u}_{\text {equation }}}{\mathbf{u}_{\text {equation }}}
$$

With this definition, a percentage of error will be established to measure the differences between one solution and another. Now we can compute some geometries like the following ones :

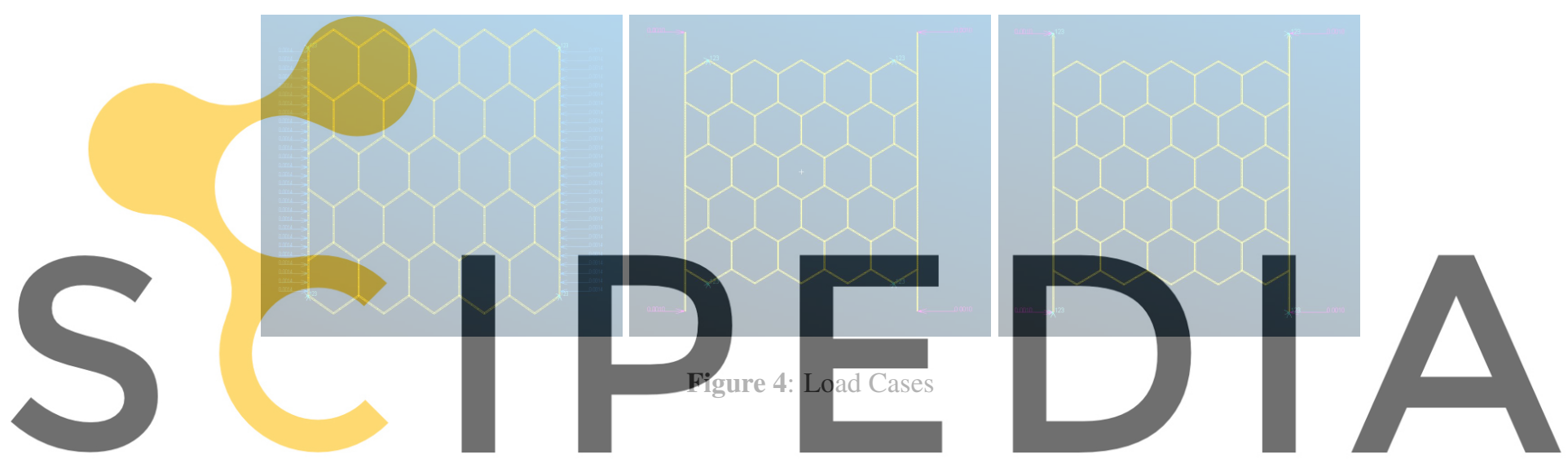

The results for these cases are shown below, the displacements being those of the upper node of the

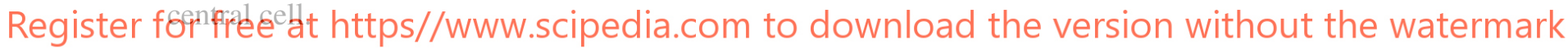

Table 1: Load cases

\begin{tabular}{llll}
\hline Load & u nastran $(\mathbf{m m})$ & u equation $(\mathbf{m m})$ & Error $(\boldsymbol{\%})$ \\
\hline Left & $3.9362 \cdot 10^{-4}$ & $4.64 \cdot 10^{-4}$ & 15 \\
Mid & $1.9391 \cdot 10^{-3}$ & $2.2 \cdot 10^{-3}$ & 11 \\
Right & $1.5259 \cdot 10^{-3}$ & $1.6792 \cdot 10^{-3}$ & 10 \\
\hline
\end{tabular}

The following geometry will considerably increase the number of cells and it will be seen how results similar to those of the previous geometry are obtained. 

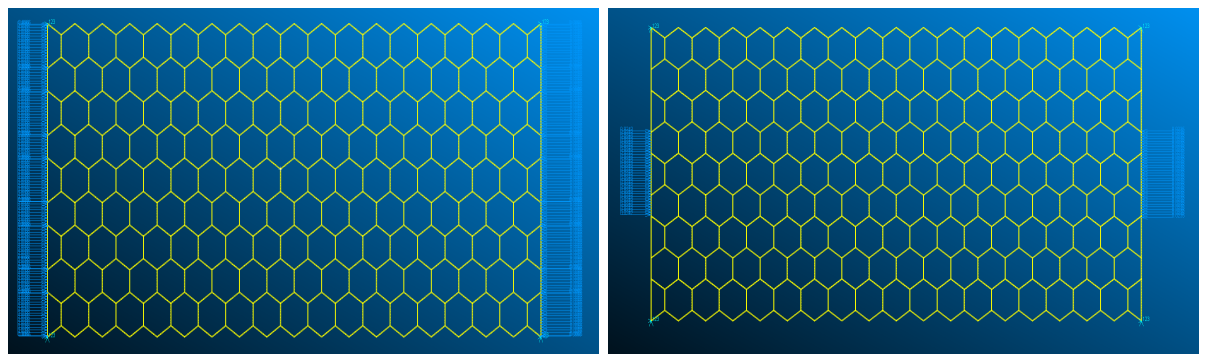

Figure 5: Load cases

For these cases are shown below, the displacements being those of the upper node of the central cell.

Table 2: Load cases

\begin{tabular}{llll} 
Load & u nastran $(\mathrm{mm})$ & u equation $(\mathrm{mmm})$ & Error $(\%)$ \\
Left & $6.87377 \cdot 10^{-3}$ & $5.948 \cdot 10^{-3}$ & 15 \\
Right & $9.4092 \cdot 10^{-3}$ & $8.5124 \cdot 10^{-3}$ & 10 \\
\hline
\end{tabular}

As has been seen, the equations predict the models to a certain degree of accuracy, since they are simple equations that take into account the action of many factors that are difficult to describe. Even so, it has been possible to study the influence of the number of cells, their arrangement, the boundary conditions
and the way to apply the load. All of this knowledge will be helpful in contipuing and implementing
virtual testing on test specimens.
3 CREATION OF TEST SPECIMIENS

There are many similarities between metamaterials and composite materials. When choosing the tests to

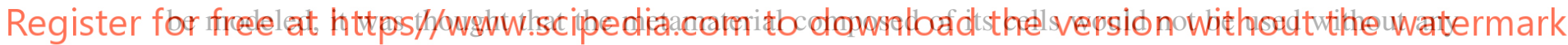

type of reinforcement or joining element with the structure that it wants to support. This is why our test tubes contain an upper and lower sheet that does the interface work, connecting the structure with the object that it wants to support. Joining this idea to the previous one mentioned, of the similarities that they contain with respect to the core of the composite material with the geometry of cells, it was decided to use a norm for composite materials as a guide. This norm contains the study tests and equations with which to estimate the properties of our metamaterials.

First, it should be stated that ASTM C 393 will be used as a guide. This means that not all the requirements that it establishes will be met. This is because in the first place, the standard is not for metamaterials and for these there are still no requirements or study methodologies for their certification. Furthermore, this is restrictive in aspects such as geometry. From a functional point of view, in these virtual tests it is not so important that the specimen is more or less large, but if you want to recreate the virtual tests with real specimens, it is important that these are not excessively large. This avoids unnecessary manufacturing costs. For this reason, a compromise solution will be reached taking into account both factors, compliance with the standard and the feasibility when reproducing the tests physi- 
cally. Factors related to the testing machine will not be addressed in this area, since the measurements are taken directly from the results provided using the MEF, without errors related to the way of measuring or measuring devices.

Various tests can be carried out to see how the materials behave and depending on which ones are made, observe some properties or others. For this, the standard intelligently chooses and modulates the conditions of two similar tests but that serve to see how the material behaves in different states of load. These tests are the three and four point bending test.

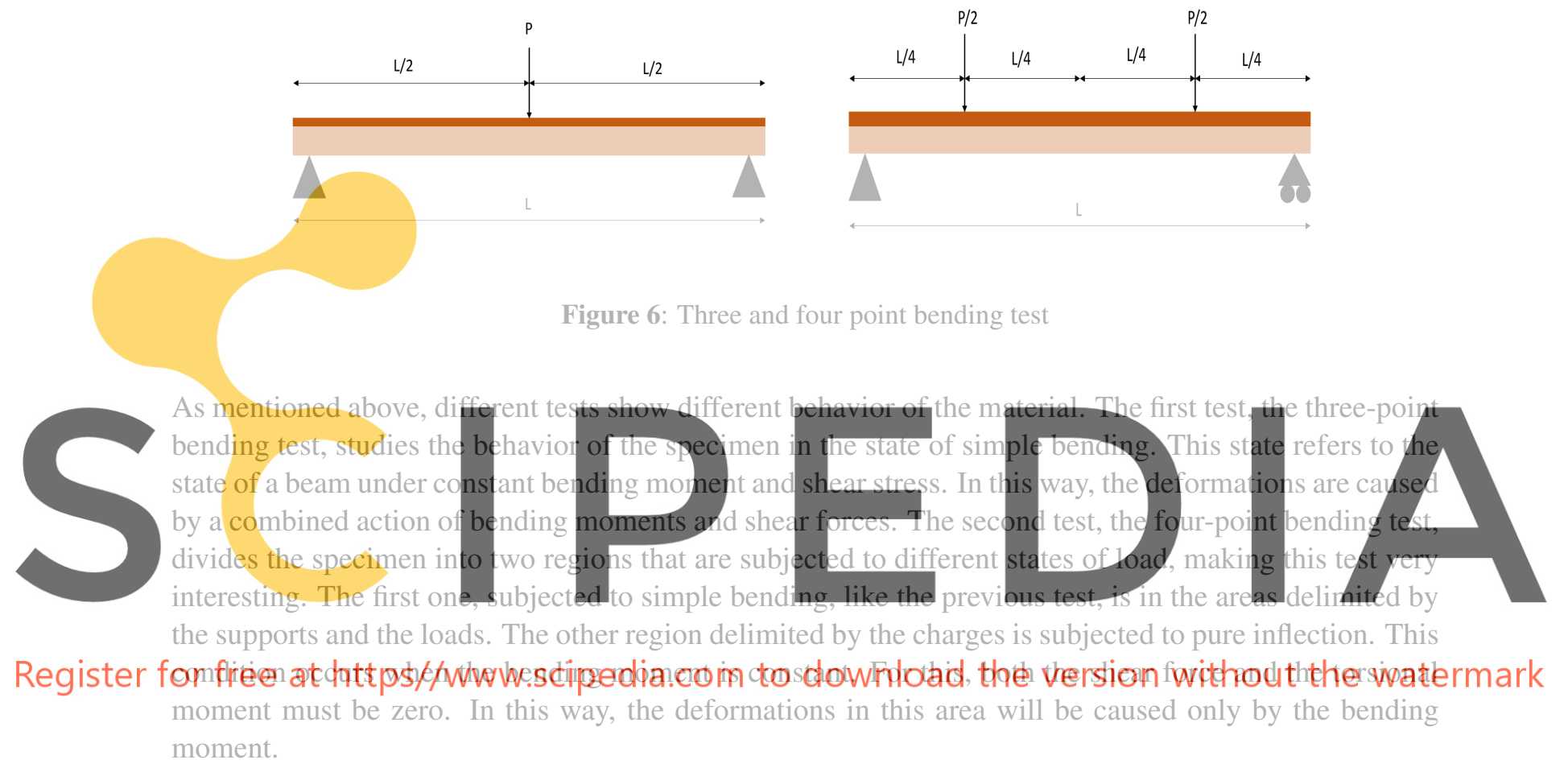

For computing the properties, the equations of the displacements from the norm ASTM C 393 will be used :

$$
\delta=\frac{\mathbf{P} \cdot \mathbf{L}^{3}}{48 \cdot \mathbf{D}}+\frac{\mathbf{P} \cdot \mathbf{L}}{\mathbf{4} \cdot \mathbf{U}}
$$

for the three-point bending test,

$$
\delta=\frac{\mathbf{1 1} \cdot \mathbf{P} \cdot \mathbf{L}^{3}}{\mathbf{7 6 8} \cdot \mathbf{D}}+\frac{\mathbf{P} \cdot \mathbf{L}}{\mathbf{8} \cdot \mathbf{U}}
$$

for the four point bending test, where $\mathrm{P}$ is the load, $\mathrm{L}$ the lenght of the test specimen, $\mathrm{U}$ the shear stiffness and D stiffness at bending moment.

In this way the norm predicts a displacement. The way to proceed when calculating the properties of the metamaterial will be as follows. A substrate material will be chosen from which the skins and the beams of the cells will be formed. With Nastran, the model adjusted to the norm will be carried out with the 
aforementioned and a displacement will be measured that included in equations of the norm will result in an estimate of the properties of the metamaterial, in this case, the elastic modulus. Within this estimate it is worth mentioning that the contribution of the upper to lower hides is included. In other tests such as those of traction or compression that could be carried out without these skins, the properties of the metamaterial will vary due to the lack of rigidity provided by the skins.

\subsection{Choice of unit cells}

The cells that are replicated will be the main variable that modulates the behavior of the metamaterial. Although the thickness of the bars that form them is also important, the internal geometry will be the cause of the greater or lesser rigidity of the metamaterial.The cells to be chosen had to be replicable in a relatively simple way, avoiding generation mechanisms such as Voronoi, since they were to be carried out with the Patran interface, which limits to moving and replicating geometric structures that follow a pattern. Therefore, the chosen cells can be contained within a cube that replicates in space, interconnecting cells. The easiest way to observe this is by looking at the cells.

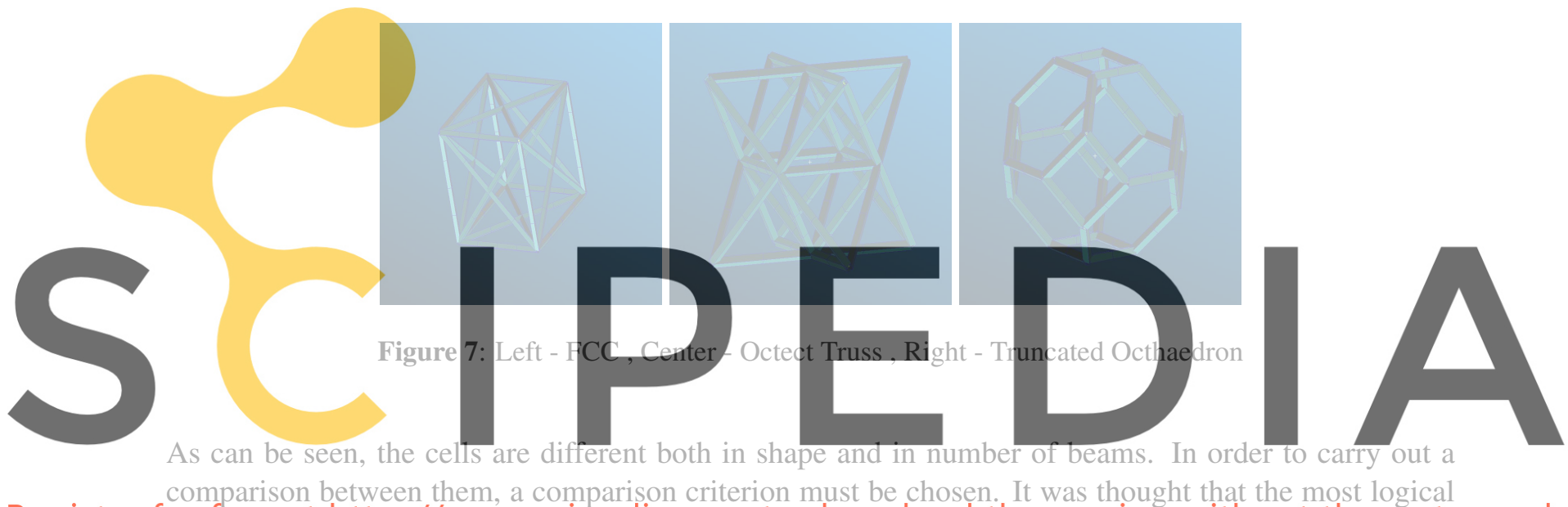

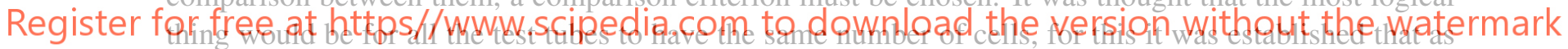

all cells can be contained in a cube, this cube would be the same size. If, on the other hand, it had been

chosen that all the bars of the celis had the same length, in some test tubes there wouid be a very different number of cells. In this way the number of cells is the same, with different bar lengths.

In addition to the thickness parameter, the volume fraction that measures the amount of space that is filled with material from the cell itself will be taken into account.

\subsection{Creation in Nastran}

With these cells they were replicated in all directions of space, creating specimens of $300 \times 28 \times 28 \mathrm{~mm}$ in the core of the specimen and with a thickness of the skins of $5 \mathrm{~mm}$. The thickness of the skins was a determining factor since a sufficient thickness is needed to be able to pass the loads into the core. Regarding the finite element model, the bars of the cells are 1D elements with the Nastran beam property with a square section and the skins are $2 \mathrm{D}$ elements with the shell property.

The union between the core and the leathers will be through a glue contact through which they will be glued, avoiding overlaps or displacements that make the core interface penetrate over the skin and vice versa. 
Regarding the boundary conditions, displacements in the areas that simulate the supports in the testing machine will be avoided without becoming embedment conditions since the longitudinal displacements are left free on one of the two sides. Finally a uniform load will be imposed that will depend on the type of test as shown in figure 6. The test specimens for the different cells in Nastran are as follows :
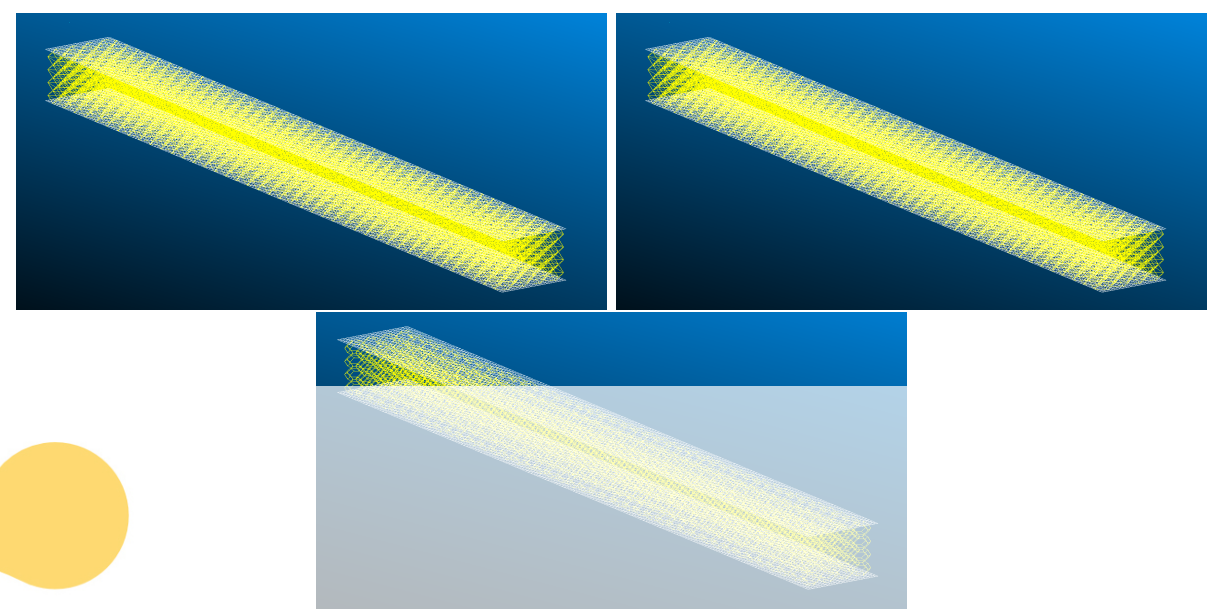

Figure 8: Test specimens in Nastran
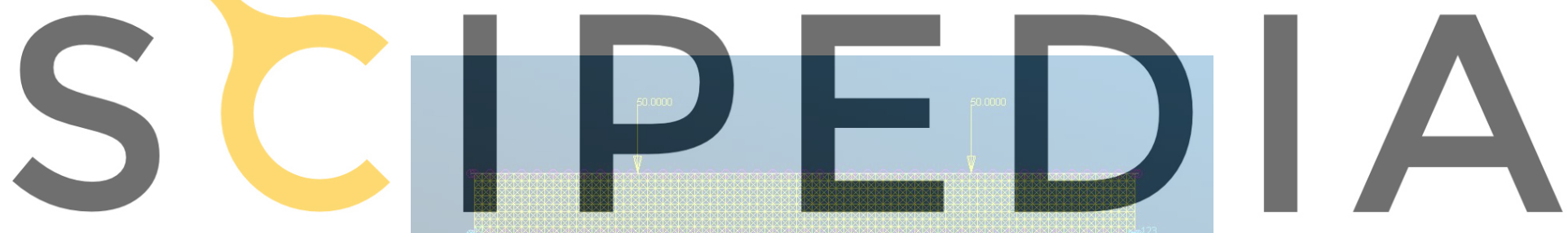

Register for free at https//www.scipedia.com to download the version without the watermark

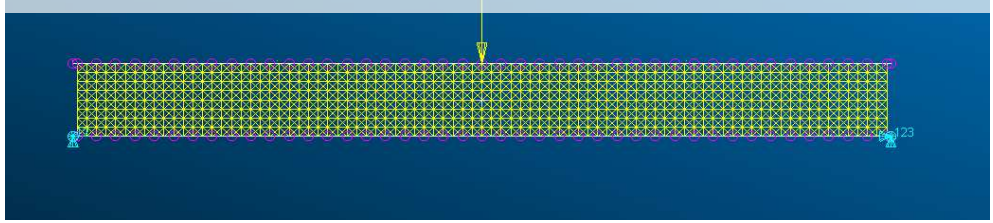

Figure 9: Test specimens in Nastran with loads and boundary conditions

\section{RESULTS}

To validate the results presented below, the composite beam was used, a beam with a double $\mathrm{T}$ section that tries to recreate the behavior of the metamaterial specimen using its elastic modulus. This study provided a field of displacements practically analogous to that of the metamaterial specimen. In addition, the non-linear solver was used looking for the effect of large displacements or deformations and different 
non-linear effects, seeing that it was unnecessary since we were in the linear regime.
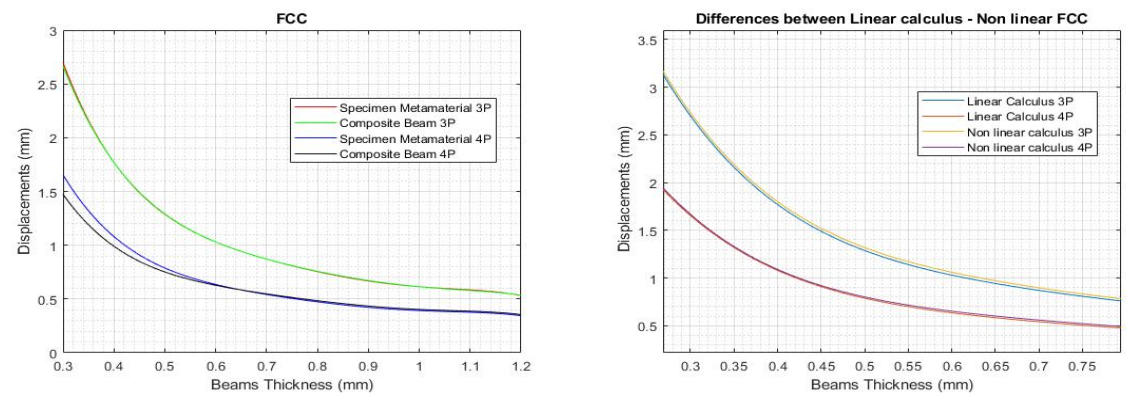

Figure 10: Composite beam verification

\subsection{Perfect and Imperfect Test Specimen}

The importance of the relative density parameter becomes clear at this time. As will be seen in the figure below when doing a linearized comparison. For the same thickness of beams, the relative densities can be very different, as for the Truncated Octahedron whose greatest beam thickness barely reaches $45 \%$ relative density. Furthermore, when introducing this parameter, a change is seen in the perception of the cells. If before the FCC cell was always lower in terms of Elastic Modulus with respect to the Octect

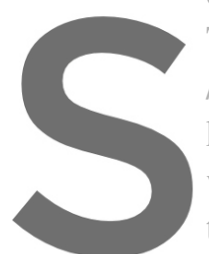
Truss, now the paradigm changes and it seems that the FCC cell is more efficient in terms / weight. As you can see, the red line in the upper figure corresponding higher. This indicates that if we are going to design all the cells wi would be convenient in terms of obtaining better properties would be the increased werght that this entails. On the other

FCC cell is more efficient. This highlights the multitude of variables involved in the problem, and the Register for free at https//WWW.Scipedia.com to download the version without the watermark
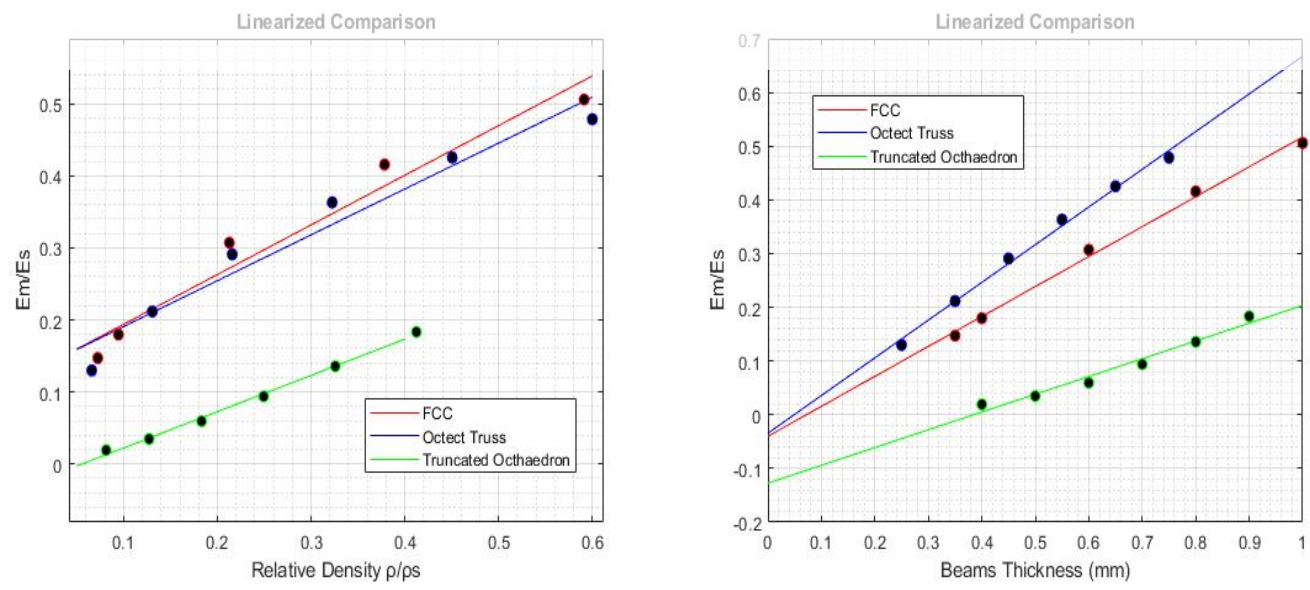

Figure 11: Linear comparison 

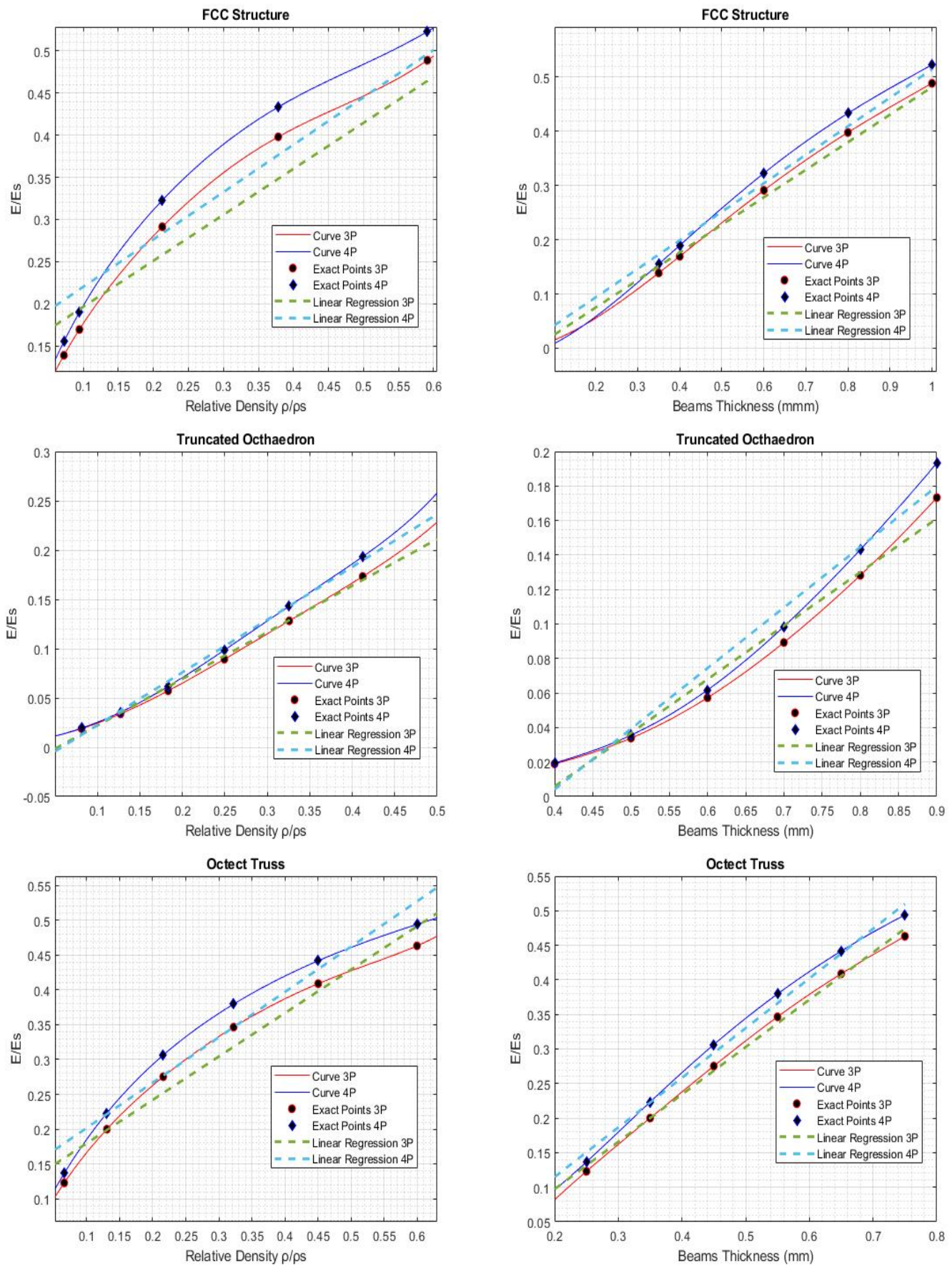

Figure 12: Test specimen 
As can be seen, different cells give different elastic modules showing how some cells are more rigid than others. Furthermore, when a manufacturing error is introduced, the elastic modulus decreases drastically, so it must be taken into account when analyzing metamaterials. In addition to the relative density, since for different applications it may be more useful to use a metamaterial with a lower elastic modulus for the same thickness of the cells, but which is more optimal from the point of view of weight.

\section{CONCLUSIONS}

Metamaterials are still growing and there is still a long way to go, both from a design and a manufacturing point of view. As was seen before any small defect can cause these structures to change their behavior a lot, so special care must be taken when manufacturing and designing them. Although the moment of manufacture is a critical moment, requiring a separate study, the entire preliminary phase of design and virtual testing to which this document is dedicated is also very important. Correctly recreating this type of structure using professional software such as Nastran and validating that the job is well done is not an easy task. As already mentioned, there are no rules or equations that validate the models, in addition to the fact that the solutions provided by the Finite Elements must be verified by the person who uses them, and cannot be considered as an absolute truth.

\section{REFERENCES}

[1] M.F Ashby, The properties of foams and lattices. Philosophical Transactions of The Royal Society, Vol. 364, pp. 1530, 2012.

[2] H. Yazdani Sarvestani, A.H. Akbarzadeh, A. Mirbolghasemi, K. Hermeneank ,3D printed metasandwich structures: Failure mechanism, energy absorption and multi-hit capability.. Materials and Design Vol.160, pp. 179-193, 2018.

[3] Georgios Maliaris, Mechanical and fracture behaviour of cellular materials withregular and random lattice structures under various compressive velocities. 4thInternational Conference of Engineering Against Failure (ICEAF IV).24-26 June 2015.

[4] Xianglong Yu, Ji Zhou, Haiyi Liang, Zhengyi Jiang and Lingling Wu, Mechanical metamaterials associated with stiffness, rigidity and compressibility : A brief review. ELSEVIER. Progress in Materials Science 94, 114-173.

[5] James Utama Surjadi, Libo Gao, Huifeng Du, Xiang Li, Xiang Xiong, Nicholas Xuanlai Fang and Yang Lu, MMechanical Metamaterials and Their Engineering Applications. Aem Journal.Advanced Engineering Materials 2019.

[6] ASTM Flexural Properties for Sandwich Structures - C939. 